\title{
Simulation and economic analysis of bovine sex selection
}

\section{Renato Travassos Beltrame ${ }^{1}$, Celia Raquel Quirino², Luís Gustavo Barioni ${ }^{3}$, Vera Fernanda Martins Hossepian de Lima ${ }^{4}$}

\footnotetext{
${ }^{1}$ Departamento de Medicina Veterinária, Centro Universitário do Espírito Santo - UNESC.

${ }^{2}$ Laboratório de Reprodução e Melhoramento Genético Animal, Universidade Estadual do Norte Fluminense (UENF).

${ }^{3}$ Embrapa Informática.

${ }^{4}$ Universidade Estadual de São Paulo - Campus Jaboticabal.
}

\begin{abstract}
A simulation model implemented in the programming software Delphi $\mathrm{XE}^{\circledR}$ was applied to evaluate sex selection in bovine. The hypothesis under investigation was that a dynamic model with stochastic and deterministic elements could detect the sexed semen technique to minimize pregnancy cost and to determine the adequate number of recipients required for in vivo (ET) and in vitro embryo production (IVP) in the proposed scenarios. Sex selection was compared through semen sexed using flow cytometry (C1) and density gradient centrifugation techniques (C2) in ET and IVP. Sensibility analyses were used to identify the adequate number of recipients for each scenario. This number was reinserted into the model to determine the biological and financial values that maximized ET and IVP using sexed semen (C1M and C2M). New scenarios showed that the density gradient technique minimized pregnancy cost based on the proposed scenarios. In addition, the adequate number of recipients $(\mathrm{ET}-\mathrm{C} 1 \mathrm{M}-115$ and $\mathrm{C} 2 \mathrm{M}-105) /(\mathrm{IVP}-\mathrm{C} 1 \mathrm{M}-145$ and $\mathrm{C} 2 \mathrm{M}-140)$ per donor used was determined to minimize the pregnancy cost in all scenarios.
\end{abstract}

Key Words: beef cattle, in vitro fertilization, modeling, stochastic simulation

\section{Introduction}

Reproductive efficiency has a significant impact on the economic viability in the beef and milk production industries. Nevertheless, there are few farms that take advantage of reproductive technologies, and even fewer show economically promising results (Johnson, 2005).

The control of male or female sex in animals allows for genetic progress, high productivity per unit area and flexibility of the production system by the ability to select the desired sex (Rath et al., 2009). However, the early choice of sex should be effective and efficient and result in fertility that is similar to that achieved with the conventional methods. It should also be affordable, allowing for extensive application (Hohenboken, 1999; Madalena et al., 2004).

The use of sexed semen for in vivo (ET) (Baruseli et al., 2007; Hayakawa et al., 2009) or in vitro embryo production (IVP) (Lu et al., 1999; Wilson et al., 2006; Wheeler et al., 2006; Blondin et al., 2009; Xu et al., 2009) indicates a fundamental interaction between the variables and the produced results. Therefore, blastocyst production and/ or pregnancy rates must generate a substantial economic return.

There has been a noticeable development of sex selection techniques in recent years. Several papers have been written on both ET (Baruseli et al., 2007; Hayakawa et al., 2009) and IVP (Lu et al., 1999; Wilson et al., 2006; Wheeler et al., 2006; Blondin et al., 2009; Xu et al., 2009). These studies primarily describe the reproductive benefits of these technologies, which encompass strategies exhibiting positive correlation in synchronization methods, strategies for obtaining sexed semen and differentiated treatment of the donor that produces higher blastocyst numbers and/or pregnancy rates.

However, the effect of sex selection on ET and IVP has not been formally analyzed through stochastic simulation. Therefore, the objective of this study was to evaluate the sex selection impact on the bioeconomic results of ET and IVP techniques in cattle. The hypothesis was that a dynamic model with stochastic and deterministic elements could identify the sex selection technique that most substantially minimized the pregnancy cost and reduced the ideal number of recipients for the proposed scenarios.

\section{Material and Methods}

A simulation model previously described in Beltrame (2010) and developed or the programming software Delphi $\mathrm{XE}^{\circledR}$ (Embarcadero Technologies, 2010) was used to evaluate bovine sex selection. 
The scenario of prostaglandin administration in recipients of ET and IVP was used. Five donors were used in each collected or aspiration procedure with a simulation time of ten years and a collection or aspiration interval of 60 days for ET and 30 days for IVP.

The following technical parameters were assigned to the model in this study: method of synchronization of recipients based on prostaglandin, herd estrus rate of $70 \%$; recipient synchronization rate of $70 \%$; and transferred/ treated recipient rate of $60 \%$, with the total recipients ready to receive an embryo.

Scenarios C1 and C2 present the pregnancy rate in nonfrozen embryo, unfreezing embryo pregnancy rate, and acuity rate (male/female rate) related to the technique that was used to obtain sexed sperm (Table 1).

Monthly recipient costs were inserted into the model according to five categories (adaptation - US\$ 39.13; recipients ready to synchronize - US\$ 21.38; recipients ready to receive an embryo - US\$ 35.13; recipients awaiting pregnancy diagnosis - US\$ 21.38; recipients after pregnancy diagnosis - US\$ 24.06), which estimated all possible recipient statuses in activity. In addition to these values, data was also used for the purchase and residual value of the donor (US\$ 9375.00), slaughter price of the recipient (US\$ 612.50), cost of maintenance of the donor (US\$ 30.74), cost of the superovulation protocol (US\$ 189.50), embryo production cost (IVP) (US\$ 43.75), honorarium on ET (US\$ 312.50) and aspiration (US\$ 187.50) and non sex selection price (US\$ 1562.50) and sexing male (US\$ 1562.50) or female (US\$ 2500.00). Other estimates and additional financial information are described in Beltrame (2010). Mortality, climatic variables and nutritional variables were not considered in the present study and will be investigated in future studies.

The estimates inserted into the model were used to determine the economic indicators, net present value (NPV) and internal rate of return (IRR), as well as to indicate the adequate number of recipients and to determine the most effective sex selection technique in the proposed scenarios.

Table 1 - Reproductive rates considered in sex selection using flow cytometry $(\mathrm{C} 1)$ and density gradient centrifugation (C2) for in vivo (ET) and in vitro (IVP) embryo production

\begin{tabular}{lcccc}
\hline Sexing & \multicolumn{2}{c}{ ET } & \multicolumn{2}{c}{ IVF } \\
\hline Method of sexing/rates & C1 & C2 & C1 & C2 \\
Pregnancy rate (\%) & 35 & 50 & $27(3)$ & 40 \\
Unfreezing pregnancy rate (\%) & 20 & 25 & 20 & 25 \\
Acuity rate & 85 & 65 & 85 & 65 \\
\hline
\end{tabular}

Source: Lu et al., 1999; Wheeler et al., 2006; Wilson et al., 2006; Hossepian de Lima, 2007; Blondin et al., 2009; Hayakawa et al., 2009; Lucio et al., 2009; Resende et al., 2009; and Xu et al., 2009.
A social discount rate of $6 \%$ was chosen to reflect actual situations based on the annual income from savings accounts.

The results obtained through simulation usually consider variation due to the technique. The problems due to variability in the number of embryos recovered or produced by follicular aspiration, the number and length of time before recipients are subjected to the procedures and freezing of the embryos directly influence the pregnancy cost. It is necessary, therefore, to define a criterion for determining the optimum point to make a decision. In this study, this criterion was the cost per pregnancy. The adequate number, based on the technique of sex selection, would be the number of recipient for which cost per pregnancy was minimal for each scenario and each reproductive technique.

For the purposes of model development and to ensure that the cost was similar to reality, it was assumed that all donors were consecutively superovulated and the samples were collected 3 times and/or were aspirated during a similar period (6 months); it was also assumed that these steps preceded pregnancy.

The production of the sexed embryos was represented in ET and IVP through stochastic simulation and negative exponential distribution (Beltrame et al., 2009, 2010a). The parameter distribution was adjusted to produce an average of 4.2 embryos per collection or aspiration (Baruselli et al., 2007). No correlations were considered between the collections from the same donor, and all fetuses were sexed using ultrasound at the time of pregnancy diagnosis without additional cost.

The model can be mathematically expressed as:

$$
G i=I f . \alpha_{g f}+I c . \alpha_{g c}
$$

Where: $G i$ is the number of pregnancies obtained per collection, or aspiration, If is the number of transference with fresh embryos and $I c$ is the number of transference with frozen embryos. Parameters $\alpha_{g c}$ and $\alpha_{g f}$ are pregnancy rates of ET or IVP embryos transferred fresh and postthawing, respectively.

$$
I c=-\operatorname{Min}(\triangle C i, 0)
$$

Where $\Delta C i$ is the variation in the stock of frozen embryos, defined as:

$$
\Delta C i=\operatorname{Max}((\text { Eet }+ \text { Eivf })-I i,-C i)
$$

Where $\Delta \mathrm{Ci}>0$ indicates that there was freezing and $\Delta \mathrm{Ci}<0$ indicates that there was transference.

Since the number of embryos collected cannot be negative, the distribution used is truncated by the Max function (which returns to the maximum value between two arguments), so that Eet and Eivf values are always equal to or greater than zero. 
The sperm sexing was modeled through the number of blastocysts obtained through collection or aspiration, assuming fertilization with dose of semen enriched with sperm from the sex required. It can be mathematically expressed by the following equation:

$$
B_{S e x}=B i \cdot \alpha_{m}+B i \cdot \alpha_{f}
$$

Where: $B_{S e x}$ is the number of sexed blastocysts; $B i$ is the number of blastocysts; $\alpha_{m}$ is the probability of blastocysts being male; and $\alpha_{f}$ is the probability of blastocysts being female.

Simulations were performed 5000 times to establish the average number of pregnancies and the costs for each scenario. Sensitivity analysis of the number of recipients was performed in each case in an attempt to identify the optimal number of recipients per donor. The appropriate number of recipients was inserted into the model to determine the biological and financial ratios that optimize the ET and IVP through the use of sexed semen. These new scenarios were referred to as $\mathrm{C} 1 \mathrm{M}$ (flow cytometry) and C2M (density gradient) at both ET and IVP.

Statistically, the analysis of variance of the number of pregnancies was used to verify that there were differences between the simulated scenarios. The average numbers of pregnancies generated were compared using the SNK test (Statistical Analysis System, version 9.2). Based on these results, an extrapolation was used for the cost comparisons.

\section{Results}

In ET, almost all the simulated scenarios were not viable when 200 recipients were used. In three of the four scenarios, negative values were obtained for NPV (Table 2). Based on the analysis of the optimized scenarios (C1M and $\mathrm{C} 2 \mathrm{M}$ ), it is possible to detect the effect of variability on the proposed situations. In ET, when sexed semen for donor insemination was evaluated $(\mathrm{C} 1, \mathrm{C} 1 \mathrm{M}, \mathrm{C} 2, \mathrm{C} 2 \mathrm{M})$, only $\mathrm{C} 2 \mathrm{M}$ (sexing by density gradient) was viable, which indicates that the adequate number of recipients suggested by the developed model was used.

Based on the analysis of IVP (Table 3), the cost per pregnancy was US\$1698.29 when sperm sexed with the flow cytometry technique was used (C1M). In a similar scenario, sperm sexed with the density gradient technique could lead to a pregnancy that is $22 \%$ cheaper than that with CM1.

Concerning the aspects of the scene graph $\mathrm{C} 2 \mathrm{M}$ (density gradient) in IVP (Figure 4), no relationship between the minimum cost of pregnancy, the total number of pregnancies, female pregnancies and the products obtained by thawing is observed. Above a rate of 30 recipients per donor used, increases in the described variables do not contribute to decreases in the cost of pregnancy.

Table 2 - Results of the simulation model for the projection of in vivo production over ten years, considering the flow cytometry (C1 and $\mathrm{C} 1 \mathrm{M})$ and density gradient (C2 and $\mathrm{C} 2 \mathrm{M})$ sex selection techniques

\begin{tabular}{|c|c|c|c|c|}
\hline \multirow{2}{*}{$\begin{array}{l}\text { ET } \\
\text { Sexing methodology }\end{array}$} & \multicolumn{4}{|c|}{ Basic scenario - $\mathrm{PGF}_{2 \alpha}$} \\
\hline & $\mathrm{C} 1$ & $\mathrm{C} 1 \mathrm{M}$ & $\mathrm{C} 2$ & $\mathrm{C} 2 \mathrm{M}$ \\
\hline Number of donors & 5 & 5 & 5 & 5 \\
\hline Number of recipients & 200 & 115 & 200 & 105 \\
\hline Number of females & $365 \mathrm{c}$ & $381 \mathrm{~b}$ & $390 \mathrm{a}$ & $381 b$ \\
\hline Number of pregnancies & $429 d$ & $448 \mathrm{c}$ & $600 \mathrm{a}$ & $586 \mathrm{~b}$ \\
\hline Mean cost per pregnancy (US\$) & 3768.23 & 1816.36 & 2848.04 & 1446.94 \\
\hline (NPV) - sexed (US\$) & -871478.91 & -38816.78 & -753075.88 & 87272.10 \\
\hline
\end{tabular}

Means followed by same letters in the rows do not differ based on the SNK test at $5 \%$ probability.

NPV - net present value.

Table 3 - Results of the simulation model to project in vitro production (IVP) in farms, considering ten years and sex selection with the flow cytometry $(\mathrm{C} 1$ and $\mathrm{C} 1 \mathrm{M})$ and the density gradient $(\mathrm{C} 2$ and $\mathrm{C} 2 \mathrm{M})$ techniques

\begin{tabular}{|c|c|c|c|c|}
\hline IVP & & Base $\mathrm{s}$ & $\mathrm{PGF}_{2 \alpha}$ & \\
\hline Sexing methodology & $\mathrm{C} 1$ & $\mathrm{C} 1 \mathrm{M}$ & $\mathrm{C} 2$ & $\mathrm{C} 2 \mathrm{M}$ \\
\hline Number of donors & 5 & 5 & 5 & 5 \\
\hline Number of recipients & 200 & 145 & 200 & 140 \\
\hline Number of females & $657 \mathrm{~b}$ & $583 d$ & $714 a$ & $632 \mathrm{c}$ \\
\hline Number of pregnancies & $773 \mathrm{c}$ & $687 \mathrm{~d}$ & $1098 \mathrm{a}$ & $972 b$ \\
\hline Mean cost per pregnancy (US\$) & 2204.31 & 1698.29 & 1728.81 & 1326.54 \\
\hline (NPV) - sexed (US\$) & -360945.69 & 24589.63 & -145418.62 & 264876.85 \\
\hline
\end{tabular}

Means followed by same letters in the rows do not differ based on the SNK test at $5 \%$ probability.

NPV - net present value. 


\section{Discussion}

Using the model, it was possible to compare the sexed semen obtained with flow cytometry (C1) and density gradient centrifugation (C2) in both ET and IVP. Consistent with the findings described by Beltrame et al. (2010a), IRR as the economic indicator was not adequate.

The results suggested that the use of sperm sexed either with the flow cytometry or the density gradient techniques does not reduce the cost of pregnancy and therefore does not achieve a lower estimative cost. The number of pregnancies obtained at the end of the simulation by incorporating the sexing of sperm into the model is considerably lower compared with traditional techniques that are used in ET and IVP scenarios (Beltrame et al., 2010a,b). Decreased productivity is similarly mentioned in the work of Madalena et al. (2004) and Rath et al. (2009).

Aspects of the viability of sexed semen were also studied by Ruvuna et al. (1992) and Madalena et al. (2004). In these studies, the NPV was used to evaluate the use of sexed semen in AI and ET, which showed promising expectations for the implementation of this biotechnology.

Simulations performed by Madalena et al. (2004) showed the relationship between the value of sexed sperm doses and the difference between the amounts paid for the products. Although this study was performed with AI only, those authors suggested that the reduction in the fertility rate by sexed sperm compared with conventional sperm could negatively influence the estimates of the projected NPV. This assumption was confirmed in the present study because a smaller number of pregnancies were obtained when the sexed sperm was used in ET and IVF.

In cases in which sperm sexing is used, two factors are crucial for the viability of the activity: the number of pregnancies and the additional amount paid due to the confirmation of the pregnancy of the desired sex (Madalena et al., 2004). Ruvuna et al. (1992) found major increases in the proportion of males when using sexed sperm to produce bulls. Smeaton \& Vivanco (2002) noted that the value of sexed semen in ET beef cattle was dependent on the cost paid for males and females at birth

In traditional IVP, the analysis of the economic indicators is based on fetal sexing with ultrasound at the diagnosis of the pregnancy (Beltrame et al., 2010a,b), but the same results are not obtained with sex selection in IVP. When using sexed semen, the ultrasound technique is mainly used to confirm the gender chosen.

Based on the analysis of IVP (Table 3), expensive pregnancies could be due to the reduction of the number of blastocysts (Blondin et al., 2009). Consequently, the number of pregnancies combined with the number and length of time before recipients are subjected to the procedures at the end of the simulation has a direct impact on the increase in the cost of pregnancy.

Comparing the optimized scenarios in which sexed semen is used (Tables 2 and 3), superiority in the total number of pregnancies is observed in the scenario based on the semen produced with the density gradient technique. As reported by some authors (Andersson et al., 2006) and used in this research, we suggest a reduction in the pregnancy rates when sperm is sexed with the flow cytometry technique.

Although several authors have reported an economic benefit of using sexed sperm (Taylor et al., 1988; Ruvuna et al., 1992; Madalena et al., 2004) in similar scenarios, due to inefficiencies of the sex selection procedure, the low number of blastocysts and the reduced pregnancy rate, reduced cost estimates of pregnancy compared with traditional techniques were not found in this study (Figure 1). In contrast, the NPV analysis confirms the findings described by Madalena et al. (2004), and allows higher incomes (as differentiated payment) because a greater number of pregnancies from the desired sex is obtained.

The assumption of fetal sexing in the elaboration of scenarios when sex selection is used was employed to eliminate failure due to the accuracy rate of the chosen technique and to precisely determine a particular sex. In this study, this technique was not responsible for any increased economic result.

For the estimate of the total pregnancies and the total number of females produced in each scenario using sexed sperm compared with in vivo embryo production (Figures 2 and 3), although there are lower rates of accuracy, evidence of a higher number of females at the end of the simulation produced with the density gradient technique is seen in both techniques. This fact is explained by the natural difference between the pregnancy rates achieved with the two methods of sexing.

This study demonstrated that the sensitivity analysis allows for the determination of the adequate number of recipients per donor. Although the number of recipients is variable and is specific for different systems, there will always be a number of recipients that will minimize the cost of pregnancy. The number of recipients in any idealized scenario and technique will be responsible for reducing the idleness in the use of the recipient, reducing embryonic waste and maximizing the production from freezing embryos (Figure 4).

Comparing the sex selection in ET and IVP, it is clear that a higher number of pregnancies was obtained using IVP in all scenarios (Table 4). This result is due to shorter intervals between aspirations and led to an increased production of calves. 


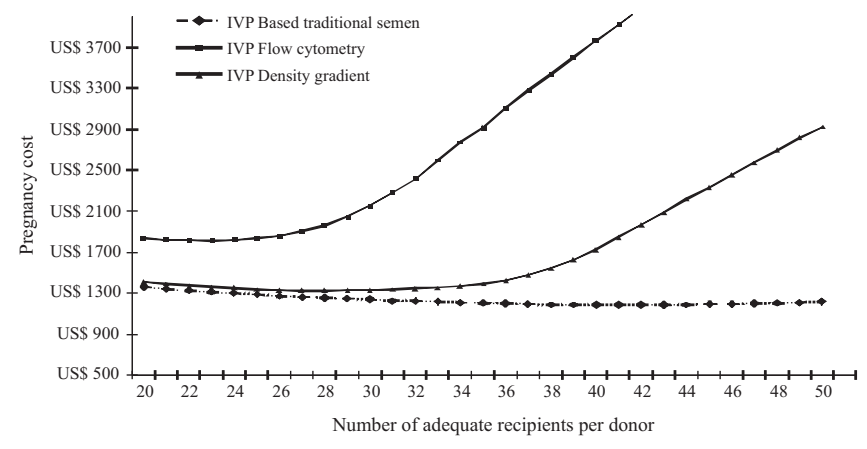

Figure 1 - Variation in pregnancy cost with traditional sperm (Beltrame, 2010) and sexed sperm during in vitro production (IVP).

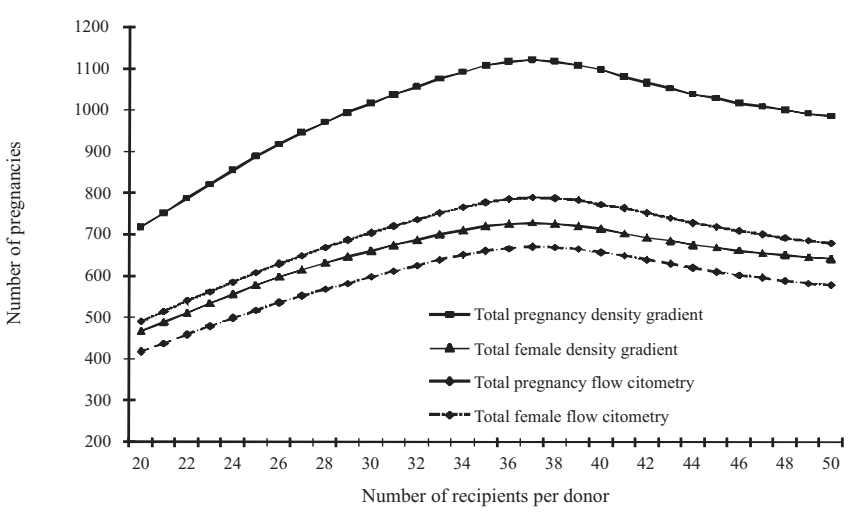

Figure 3 - Estimate of the total number of pregnancies and the total number of females produced in each scenario using sexed sperm compared with in vitro embryo production.

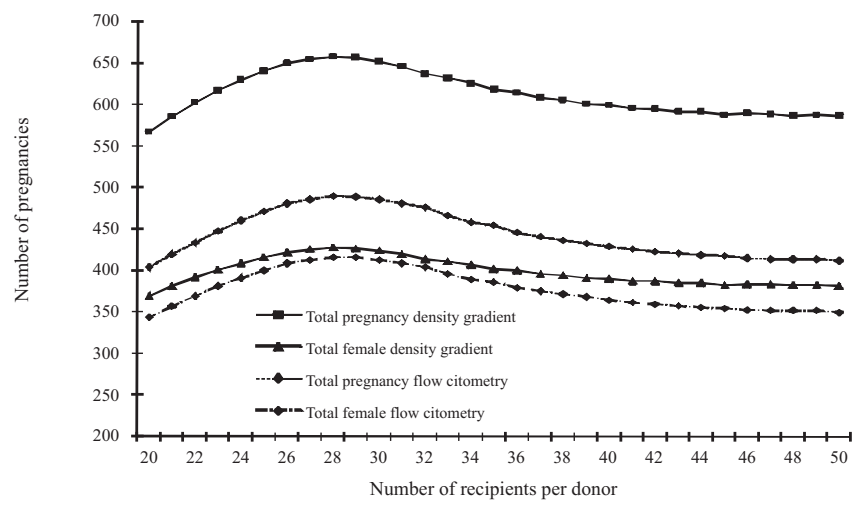

Figure 2 - Estimate of the total pregnancies and the total number of females produced in each scenario using sexed sperm compared with in vivo embryo production.

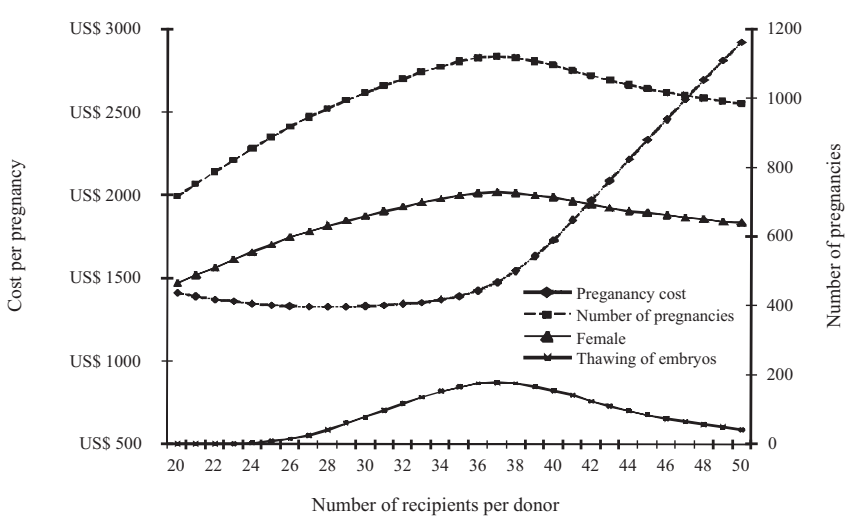

Figure 4 - Aspects of the curves of the cost of pregnancy, number of pregnancies, number of females and number of products with thawing based on the use of sperm sexed with density gradient in in vitro embryo production.

Table 4 - Comparison between number of pregnancies, number of females and cost of pregnancy for in vivo (ET) and in vitro (IVP) embryo production techniques

\begin{tabular}{|c|c|c|c|c|}
\hline \multirow{3}{*}{$\begin{array}{l}\text { ET/IVP } \\
\text { Sexing methodology }\end{array}$} & \multicolumn{4}{|c|}{ Basic scenario - $\mathrm{PGF}_{2 \alpha}$} \\
\hline & \multicolumn{2}{|c|}{ Flow cytometry - C1M } & \multicolumn{2}{|c|}{ Density gradient - C2M } \\
\hline & ET & IVP & ET & IVP \\
\hline Number of recipients & 115 & 145 & 105 & 140 \\
\hline Number of female & $380 \mathrm{c}$ & $583 b$ & $381 \mathrm{c}$ & $632 \mathrm{a}$ \\
\hline Number of pregnancy & $448 d$ & $687 b$ & $586 \mathrm{c}$ & $972 \mathrm{a}$ \\
\hline
\end{tabular}

Means followed by same letters in the rows do not differ based on the SNK test at 5\% probability.

NPV - net present value.

\section{Conclusions}

Sperm sexed with the density gradient technique minimized the cost of pregnancy in the proposed scenarios. In addition, the appropriate number of recipients for use in all scenarios was identified.

\section{References}

ANDERSSON, M.; TAPONEN, J.; KOMMERI, M. et al. Pregnancy rates in lactating holstein-friesian cows after artificial insemination with sexed sperm. Reproduction in Domestic Animals, v.41, p.95-97, 2006.

BARUSELLI, P.S.; SOUZA, A.H.; MARTINS, C.M. et al. Sexed semen: Artificial insemination and embryo transfer. Revista Brasileira de Reprodução Animal, v.31, p.374-381, 2007. 
BELTRAME, R.T.; BARIONI, L.G.; QUIRINO, C.R. et al. Probability density function of the number of embryos collected from superovulated Nelore breed donors. Scientia Agricola, v.66, p.447-450, 2009.

BELTRAME, R.T. Bioeconomic impact of sperm sexing in embryo transfer and in vitro fertilization in zebu. 2010. 113p. Thesis (Doctorate in Animal Science) - Universidade Estadual do Norte Fluminense, Campos dos Goytacazes.

BELTRAME, R.T.; QUIRINO, C.R.; BARIONI, L.G. et al. Simulation and economic analysis of in vivo and in vitro embryo production in bovine Pesquisa Agropecuária Brasileira, v.45, p.1513-1520, 2010a.

BELTRAME, R.T.; QUIRINO, C.R.; BARIONI, L.G. et al. In vivo and in vitro analysis of embryo production to nelore cows. Ciência Animal Brasileira, v.11, p.21-27, $2010 \mathrm{~b}$.

BLONDIN, P.; BEAULIEU, M.; FOURNIER, V.; et al. Analysis of bovine sexed sperm for IVF from sorting to the embryo. Theriogenology, v.71, p.30-38, 2009.

EMBARCADERO TECHNOLOGIES. Delphi XE. San Francisco, California, 2010. Available at: <http://www.embarcadero.com/br/ products/delphi>. Accessed on: Dec. 20, 2010.

HAYAKAWA, H.; HIRAI, T.; TAKIMOTO, A. et al. Superovulation and embryo transfer in holstein cattle using sexed sperm. Theriogenology, v.71, p.68-73, 2009.

HOHENBOKEN, W.D. Applications of sexed semen in cattle production. Theriogenology, v.52, p.1421-1433, 1999.

HOSSEPIAN DE LIMA, V.F.M. Methodological advances on spermatozoa sexing for using in genetic improvement and animal production. Revista Brasileira de Zootecnia, v.36, p.219-228, 2007.

JOHNSON, S.K. Possibilities with today's reproductive technologies. Theriogenology, v.64, p.639-656, 2005.

LU, K.H.; CRAN, D.G.; SEIDEL, J.G.E. In vitro fertilization with flow-cytometrically-sorted bovine sperm. Theriogenology, v.52, p.1393-1405, 1999.
LUCIO, A.C.; OLIVEIRA, L.Z.; CELEGHINI, E.C.C. et al. Influence of bovine subspecies in the recovered rate after the separation of $\mathrm{x}$-bearing sperm by centrifugation in discontinuos percoll density gradient. Animal Reproduction, v.6, p.336-336, 2009.

MADALENA, F.E.; JUNQUEIRA, F.S. The value of sexed bovine sêmen. Journal of Animal Breeding and Genetics, v.121, p.253-259, 2004.

RATH, D.; MOENCH-TEGEDER, G.; TAYLOR, U. et al. Improved quality of sex-sorted sperm: A prerequisite for wider commercial application. Theriogenology, v.71, p.22-29, 2009.

RESENDE, M.V.; BEZERRA, M.B.; PERECIN, D. et al. Sepapartion of X-bearing bovine sperm by centrifugation in continuos percoll and optiprep density gradient: effect in sperm viability and in vitro embryo production. Ciência Animal Brasileira, v.10, p.581-587, 2009.

RUVUNA, F.; TAYLOR, J.F.; WALTER, J.P. et al. Bioeconomic evaluation of embryo transfer in beef production systems: III. Embryo lines for producing bulls. Journal of Animal Science, v.70, p.1091-1097, 1992.

SMEATON, D.C.; VIVANCO, W.H. Profitability of new reproductive technologies in beef production systems. Proceedings of New Zealand Society of Animal Production, v.62, p.133-137, 2002.

TAYLOR, J.F.; PHILLIPS, K.R.; TOMASZEWSKI, M.A. Net present value and economic merit of sexed semen and splitting units of semen for Australian Holsteins. Journal of Dairy Science, v.71, p.3100-3111, 1988.

WILSON, R.D.; FRICKE, P.M.; LEIBFRIED-RUTLEDGE, M.L. et al. In vitro production of bovine embryos using sex-sorted sperm. Theriogenology, v.65, p.1007-1015, 2006.

WHEELER, M.B.; RUTLEDGE, J.J.; AMY, F.B. et al. Application of sexed semen technology to in vitro embryo production in cattle. Theriogenology, v.65, p.219-227, 2006.

XU, J.; CHAUBAL, S.A.; Du, F. Optimizing IVF with sexed sperm in cattle. Theriogenology, v.71, p.39-47, 2009. 